\title{
Ammonia monitoring at trace level using photoacoustic spectroscopy in industrial and environmental applications
}

\author{
Stéphane Schilt ${ }^{\mathrm{a}, *}$, Luc Thévenaz $^{\mathrm{a}}$, Marc Niklès ${ }^{\mathrm{b}}$, Lukas Emmenegger ${ }^{\mathrm{c}}$, Christoph Hüglin $^{\mathrm{c}}$ \\ ${ }^{a}$ Laboratory of Nanophotonics and Metrology (NAM), Swiss Federal Institute of Technology (EPFL), CH-1015 Lausanne, Switzerland \\ b Omnisens SA, Science Park, CH-1015 Lausanne, Switzerland \\ ${ }^{\text {c } E M P A}$ Dübendorf, Ueberlandstrasse 129, CH-6800 Dübendorf, Switzerland
}

Received 22 October 2003; accepted 24 November 2003

\begin{abstract}
An ammonia traces analyser based on photoacoustic spectroscopy is described. The system uses a $\mathrm{CO}_{2}$ laser and a properly designed resonant photoacoustic cell to achieve ammonia detection at sub-parts-per-billion (ppb) level. The instrument features unattended automatic on-line monitoring of ammonia with a detection limit of $0.1 \mathrm{ppb}$. Interferences from atmospheric $\mathrm{CO}_{2}$ and $\mathrm{H}_{2} \mathrm{O}$ are efficiently suppressed by a careful selection of the laser wavelength and a compensation of the water vapour signal made with a high-precision hygrometer. The cell design enables continuous measurement at high flow rates (up to $51 / \mathrm{min}$ ), which guarantees a fast response time of the system for the monitoring of ammonia, a sticky polar molecule that adheres to most surfaces. Various examples of applications of the instrument in the semiconductor industry and for atmospheric pollution monitoring are presented. They demonstrate the excellent performances of the system and its suitability for these applications.
\end{abstract}

(C) 2004 Elsevier B.V. All rights reserved.

Keywords: Photoacoustic spectroscopy; Ammonia monitoring; $\mathrm{CO}_{2}$ laser; Trace gas detection; IR spectroscopy

\section{Introduction}

Photoacoustic spectroscopy (PAS) is an extremely sensitive technique for trace gas measurements. It is based on the photoacoustic (PA) effect, which consists schematically in the generation of a sound wave in a medium irradiated by a modulated light beam. This phenomenon has been known since 1880, when Bell [1] had discovered it first in solids, and later on in gases. His discovery primarily lead to the construction of a new device for phone communication, called the "photophone", an instrument in which light produces sound [2]. The potential of this technique for spectroscopy was rapidly recognised, but a long time was necessary before the PA effect was actively exploited. In fact,

\footnotetext{
* Corresponding author. Tel.: +41-21-693-3969; fax: +41-21-693-2614.

E-mail addresses: stephane.schilt@epfl.ch (S. Schilt), luc.thevenaz@epfl.ch (L. Thévenaz), marc.nikles@omnisens.ch (M. Niklès), lukas.emmenegger@empa.ch (L. Emmenegger), christoph.hueglin@empa.ch (C. Hüglin).
}

the actual start of its application in spectroscopy is associated with two crucial discoveries made in electronics and opto-electronics: the microphone and the laser. Small electret microphones enable sensitive detection of the generated sound wave, whereas lasers are ideally suited for PAS owing to their excellent spectral purity and high power optical emission.

Trace gas monitoring systems based on PAS have been investigated by many groups all around the world [3-14]. Different types of cell configurations (non-resonant [3]; radial [4-6], azimuthal [7,8] or longitudinal [9-11] resonances of a cylindrical resonator; Helmholtz resonators $[12,13])$ and different kinds of exciting laser sources (gas lasers [4-6]; DFB semiconductor laser diodes [7-9,13]; external cavity laser diodes [10,11]; quantum cascade lasers [12,14]) have been used in laboratory systems, showing extremely good performances. However, only few PA-based systems have found commercial applications up to now. Both industrial and environmental applications of a PA-based trace gas analyser developed for sub-parts-per-billion (ppb) ammonia monitoring are reported in this paper. For these two kinds of applica- 
tions (process control in the semiconductor industry and air quality monitoring), fast, precise and highly sensitive measurement of ammonia is strongly required. Gas sensors developed for such applications must fulfil requirements that are much more stringent than for laboratory systems. The main required features are:

- high sensitivity enabling detection at ppb or sub-ppb levels;

- excellent linearity over a large dynamic range;

- short response time in order to enable a real time and continuous measurement;

- long-term stability in order to guarantee calibration-free operation;

- high selectivity to keep interferences from other species as low as possible; the main potential interferents are atmospheric gases present at relatively high concentrations, such as water vapour or carbon dioxide;

- ease of operation as a key parameter for commercial applications; in particular, the instrument must provide user friendly operation, requiring no sampling of the gas to be analysed, no maintenance and enabling unattended automatic on-line monitoring.

PAS has demonstrated the potentiality to meet all these requirements. Various aspects of this technique are discussed in this paper, including the theoretical basics of PAS. The realisation of a portable ammonia analyser (TGA300 Series) with sub-ppb sensitivity is described, and examples of its application in the semiconductor industry and for environmental monitoring are presented.

\section{Fundamentals of laser photoacoustic spectroscopy}

The generation and detection of the PA signal in a gaseous sample can be divided into four main steps [15]:

(i) Molecular absorption of photons, resulting in an excitation of the absorbing molecules to an upper energy level.

(ii) Non-radiative relaxation of the excited state (collisional relaxation). The optical energy previously absorbed is completely released as heat in the sample, thus, inducing an expansion of the gas, i.e. a local modification of its pressure.

(iii) When the laser intensity $I$ is modulated at a frequency $f=\omega / 2 \pi\left(I=I_{0} \mathrm{e}^{\mathrm{i} \omega t}\right)$, a periodic heating of the sample is produced, which leads to periodic pressure variations. Both thermal and acoustic waves are, therefore, generated in the sample.

(iv) Detection of the acoustic wave using a microphone provided that the modulation frequency $f$ falls within the audio range.

In the case of small absorptions $(\alpha \ll 1)$, the deposited heat energy $H(r, t)$ is directly proportional to the molecular absorption coefficient $\alpha$ and to the laser beam intensity $I=$ $I_{0} \mathrm{e}^{\mathrm{i} \omega t}$ :

$H(r, t)=\alpha I_{0}(r, t) \mathrm{e}^{\mathrm{i} \omega t}$.

The two independent waves generated in the sample have a completely different behaviour [16-18]: the thermal wave is a strongly damped thermal diffusion wave of very short wavelength (smaller than a millimetre), whereas the sound wave is a weakly damped acoustic wave with a wavelength in the centimetre to metre range. Therefore, the thermal wave can be detected only in the vicinity of the excitation laser beam, whereas the acoustic wave is detectable at much larger distances from the laser beam. Both thermal and acoustic waves have amplitudes directly proportional to the gas concentration, so that their detection enables the measurement of the concentration. Different spectroscopic techniques have been developed for the measurement of such waves. Methods based on the measurement of the thermal wave have been proposed and are generally identified as photothermal spectroscopy $[19,20]$. PAS is based on the detection of the acoustic wave with a sensitive microphone and has given better results so far. The present paper is focusing on PAS.

\subsection{Resonant photoacoustic spectroscopy}

The theory of the generation and detection of the PA signal in a cylindrical cell has been described in detail by several authors (see for example [15-17,21]). As the generated sound is very weak, resonant configurations are usually used. In such geometries, the PA cell is designed to be an acoustic resonator and the laser is modulated at a resonance frequency of this cavity. In such a case, constructive interferences arise between waves reflected on the cell interfaces. A standing wave is created in the resonator at a resonance frequency and its amplitude is amplified in comparison to a non-resonant configuration. One particular pressure distribution in the cell, named an eigenmode of the resonator, corresponds to each resonance frequency. For a cylindrical cavity of length $L_{\mathrm{c}}$ and radius $R_{\mathrm{c}}$, the different modes are identified by three eigenvalues $k, m$ and $n$, which represent, respectively, the longitudinal, azimuthal and radial indices. Among all the modes of the cavity, we can distinguish pure longitudinal $(m=n=0)$, pure azimuthal $(k=n=0)$ and pure radial modes $(k=m=0)$. The first two modes of each type are illustrated in Fig. 1. Other eigenvalues are associated to hybrid modes. The resonance frequency of mode $j=[k, m, n]$ corresponds to

$\omega_{j}=2 \pi f_{j}=\pi c_{\mathrm{s}} \sqrt{\left(\frac{k}{L_{\mathrm{c}}}\right)^{2}+\left(\frac{\alpha_{m n}}{R_{\mathrm{c}}}\right)^{2}}$,

where $c_{\mathrm{s}}$ is the sound velocity in the sample and $\alpha_{m n}$ is proportional to the $n$th root of the derivative of the $m$ th order Bessel function [21], whose first values are given in Table 1. 

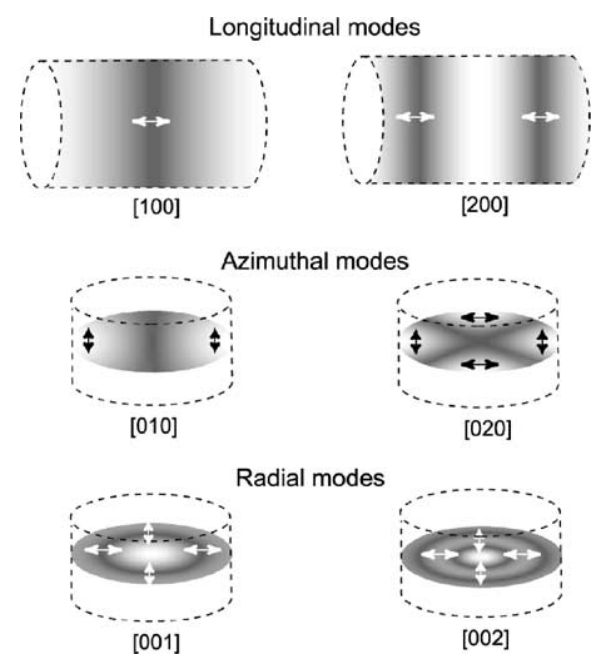

Fig. 1. First eigenmodes of a cylindrical acoustic cavity. Dark zones correspond to nodes and white zones to anti-nodes of the acoustic standing wave.

The amplitude of mode $j$ is given by

$$
\begin{aligned}
A_{j}(\omega)= & \frac{\mathrm{i} \omega}{\omega_{j}^{2}} \frac{\gamma-1}{V_{\mathrm{c}}} \frac{\alpha P_{0} L_{\mathrm{c}}}{\left[\left(1-\omega^{2}\right) / \omega_{j}^{2}\right]+\left[\mathrm{i} \omega /\left(\omega_{j} Q_{j}\right)\right]} \frac{1}{L_{\mathrm{c}}} \\
& \times \int \mathrm{d} V g(r) p_{j}^{*}(r, \omega),
\end{aligned}
$$

where $\gamma=C_{\mathrm{p}} / C_{\mathrm{v}}$ is the ratio of the specific heat at constant pressure and constant volume, $V_{\mathrm{c}}=\pi R_{\mathrm{c}}^{2} L_{\mathrm{c}}$ is the cell volume, $P_{0}$ the laser power and $Q_{j}$ is the quality factor of the resonance, that takes into account the losses in the acoustic wave. The integral $I_{j}=1 / L_{\mathrm{c}} \int \mathrm{d} V g(r) p_{j}^{*}(r, \omega)$ in the numerator of the expression of the mode amplitude $A_{j}$ represents the coupling between the laser beam profile, described by the normalised distribution $g(r)$, and the acoustic mode $p_{j}$ (overlap integral). A large overlap between the laser beam distribution and the acoustic mode results, therefore, in an efficient excitation of the PA signal. This conditions the way of coupling the laser beam into the PA cell, in order to maximize the signal.

\subsection{Excitation of the acoustic resonance}

At the resonance $\left(\omega=\omega_{j}\right)$, the PA signal detected with a microphone located at $r=r_{\mathrm{M}}$ becomes

\section{Table 1}

Numerical values of the parameters $\pi \alpha_{m n}$ for the first cylindrical Bessel functions

\begin{tabular}{lllrl}
\hline$m$ & $n$ & & & \\
\cline { 2 - 5 } & 0 & 1 & 2 & 3 \\
\hline 0 & 0 & 3.832 & 7.016 & 10.173 \\
1 & 1.841 & 5.331 & 8.536 & 11.706 \\
2 & 3.054 & 6.706 & 9.969 & 13.170 \\
3 & 4.201 & 8.015 & 11.346 & 14.586 \\
\hline
\end{tabular}

$p\left(r_{\mathrm{M}}, \omega_{j}\right)=\frac{Q_{j}}{\omega_{j}} \frac{(\gamma-1) I_{j} L_{\mathrm{c}}}{V_{\mathrm{c}}} p_{j}\left(r_{\mathrm{M}}, \omega_{j}\right) \alpha P_{0}$.

A resonant configuration produces an amplification of the PA signal by the quality factor $Q_{j}$. In the vicinity of the resonance $\left(\omega \cong \omega_{j}\right)$, the acoustic energy, which is proportional to the square of the pressure, is described by a Lorentzian distribution with a half-width at half maximum

$\Delta \omega_{j}=\frac{\omega_{j}}{2 Q_{j}}$.

Therefore, the quality factor can be determined experimentally from the measurement of the spectral distribution of acoustic energy. $Q$ values of several hundreds can be reached in properly designed resonators, resulting in an amplification of the acoustic wave of the same value.

Two terms can be distinguished in the expression of the PA signal at resonance (see Eq. (4)): the first one $\left(\alpha P_{0}\right)$ represents the optical energy absorbed in the sample, whereas the second describes the sensitivity of the PA resonator. As this latter term only depends on the cell design, it is conventionally named the cell constant and is expressed in $\mathrm{Pacm} / \mathrm{W}$

$C_{j}\left(\omega_{j}\right)=\frac{Q_{j}}{\omega_{j}} \frac{(\gamma-1) I_{j} L_{\mathrm{c}}}{V_{\mathrm{c}}} p_{j}\left(r_{\mathrm{M}}, \omega_{j}\right)$.

The cell constant depends on the geometrical dimensions of the cell, but also on the frequency and quality factor of the selected resonance, and on the overlap integral of the optical beam with the acoustic mode. As the cell constant determines directly the sensitivity of a PA system, the highest possible value should be reached for this parameter. The ways for improving the cell constant are a small cell volume $V_{\mathrm{c}}$, a low resonance frequency $\omega_{j}$, a high quality factor $Q_{j}$ and a high overlap integral $I_{j}$. However, most of these parameters are not independent. For example, a small volume results in a high resonance frequency according to (2). The quality factor is also influenced by $\omega_{j}, L_{\mathrm{c}}$ and $R_{\mathrm{c}}$. Therefore, the optimal trade-off must be thought between all these parameters in order to reach a high cell constant. This constitutes a key factor in the development of sensitive PA system.

\section{TGA300 series: realisation of a portable ammonia analyser for sub-ppb detection}

The laser-based PA trace gas analyser TGA300 series includes two models that have been designed with respect to their specific application requirements. Model TGA320 has been developed for process control in the semiconductor industry, while TGA310 has been designed for atmospheric pollution monitoring.

\subsection{Instrument design}

The instrument layout is shown schematically in Fig. 2. It consists of a laser source, a resonant PA cell, a reference solid-state detector for laser power measurement and 


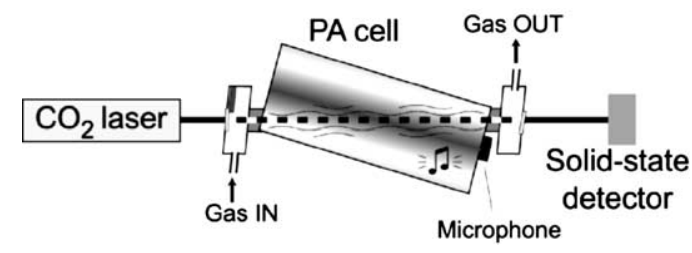

Fig. 2. Schematic representation of the $\mathrm{PA}$ instrument, using a $\mathrm{CO}_{2}$ laser, a radial PA measurement cell and a solid-state reference detector. The amplitude of the first radial mode is shown in greyscale, with the pressure nodes in white. The laser beam enters and leaves the resonator at the nodes of the radial mode.

a control electronic module for laser modulation and signal processing. The DSP-based electronics completely and autonomously controls the measurements (resonance tracking, acoustic signal processing) and computes the ammonia concentration with an updating time as short as $3 \mathrm{~s}$. A dedicated computer directly interfaces with the system and enables automatic unattended monitoring.

The laser source is a sealed-off RF-excited $\mathrm{CO}_{2}$ laser. Single-mode emission and wavelength selection is granted by an intracavity grating. The PA cell is made of a central cylindrical resonator and two buffer volumes acting as acoustic filters. The cell is operated in the first radial mode of the resonator, occurring near $10.5 \mathrm{kHz}$. The choice of a radial resonance is motivated by several parameters. First of all, a radial resonance generally offers a high quality factor, because there is no viscous damping of the acoustic wave on the side walls. As a result, the radial resonance produces a larger enhancement factor $Q$ as compared to longitudinal and azimuthal modes [22]. Furthermore, radial configurations are attractive for ammonia monitoring at low mixing ratios, because the cell windows and the gas inlet and outlet can be located on the pressure nodes of the acoustic mode, resulting in a reduction of the noise produced by window heating and gas flow [6]. Window heating contributes to the background noise (window noise) and is generally the limiting factor in PA systems operating with high power $\mathrm{CO}_{2}$ lasers [5]. The possibility of using high continuous flow rates without producing additional noise is also an advantage in the case of ammonia monitoring. Ammonia is a sticky polar molecule that adheres to most surfaces [23], making its measurement very difficult. A long rise time may be needed to reach a stable ammonia concentration in the measurement cell, as an equilibrium state between the ammonia molecules adsorbed on the cell walls and in the gas phase must be attained. However, careful selection of the cell material and the use of high flow rates yield an important reduction of rise time and guarantee a fast response.

In order to place cell windows, gas inlet and gas outlet at the pressure nodes of the radial mode, a diagonal laser beam excitation is used, as illustrated in Fig. 2. In this configuration, the microphone may be located on the cell axis, i.e. at the maximum of the pressure wave, enabling an optimum acoustic detection, which would not be possible if an axial laser beam excitation were used. In axial configu-

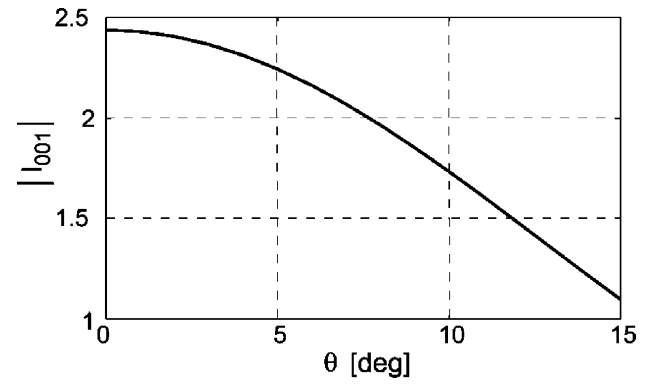

Fig. 3. Variation of the overlap integral $I_{0,0,1}$ as a function of the slope angle $\theta$ between the laser beam and the resonator axis.

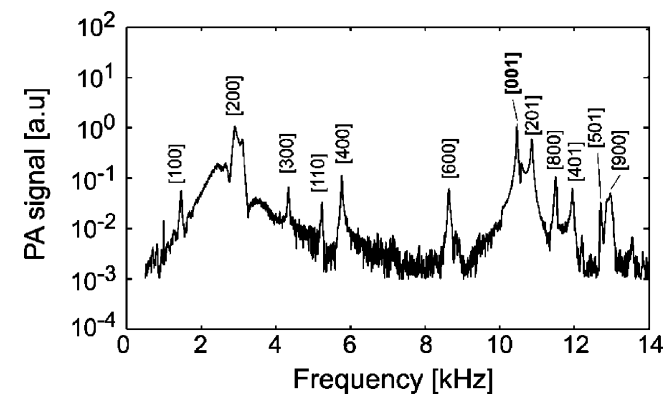

Fig. 4. Acoustic response of the PA cell. The first radial mode $[0,0,1]$ at $10.5 \mathrm{kHz}$ is best excited by the laser beam.

rations, the microphone is generally placed on the side wall of the cell [5], where the acoustic pressure is lower, resulting in a reduced sensitivity. However, the overlap integral is slightly reduced in a diagonal configuration in comparison to an axial excitation. The decrease of the overlap integral as a function of the incidence angle $\theta$ is illustrated in Fig. 3 . For the inclinaison used in the cell $\left(\theta \cong 12^{\circ}\right)$, the reduction of the overlap integral is on the order of $40 \%$. The acoustic response of the cell with this diagonal excitation is shown in Fig. 4. The first radial resonance (mode $[0,0,1]$ ) is best excited by the laser beam. This resonance is shown more in detail in Fig. 5. A fit on the experimental points with a Lorentzian distribution gives the quality factor; a value of $Q_{0,0,1}=780$ is obtained.

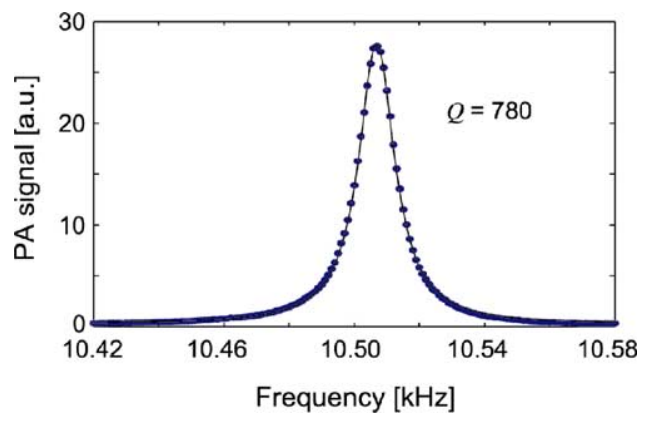

Fig. 5. Distribution of the acoustic energy in the first radial mode of the PA cell. Circles are experimental points and the curve is the result of a fit by a Lorentzian distribution. The quality factor is $Q=780$. 


\subsection{Water vapor compensation}

Ammonia detection at ppb level must be very selective to avoid the influence of other species present in the sample to be analysed. The major potential interferents are atmospheric gases such as $\mathrm{CO}_{2}$ (typically $350 \mathrm{ppm}$ in the atmosphere) and water vapour $\left(1.4 \%\right.$ at $T=23{ }^{\circ} \mathrm{C}$ and $50 \%$ relative humidity).

$\mathrm{CO}_{2}$ molecules absorb of course radiation at any $\mathrm{CO}_{2}$-laser wavelength, but the lines are relatively weak as they belong to hot bands. Interference from atmospheric $\mathrm{CO}_{2}$ can be suppressed efficiently using an isotopic $\mathrm{CO}_{2}$ laser [24] and proper wavelength selection. The mid-infrared water vapour absorption spectrum is not only made of discrete absorption lines, as every active molecule, but also presents a continuous absorption background in the range of $\mathrm{CO}_{2}$ lasers. The origin of this continuous background, named the water continuum in the literature, is not totally understood yet, but several explanations have been suggested [25]. In addition to be continuous (with a weak wavelength-dependence), this absorption shows an unusual quadratic dependence as a function of the water concentration [26], instead of the common linear dependence met in the case of a line absorption. Due to these characteristics, water absorption occurs at every wavelength and is, thus, impossible to avoid in $\mathrm{CO}_{2}$-laser PAS. Even for the strongest absorption lines of ammonia, the ambient humidity produces an interferent PA signal equivalent to several tens of ppb of ammonia. This water contribution must be subtracted from the total PA signal in order to enable ammonia detection at the ppb-level. Therefore, a compensation of the water continuum contribution has been implemented in TGA300 Series instruments. The water concentration is measured in the PA cell using a high-precision hygrometer and the corresponding PA signal is subtracted from the total measured signal. Every hygrometer response is precisely and individually calibrated with respect to the PA signal generated by known concentrations of water vapour. The error on the compensation can be reduced to less than $0.2 \%$, leaving an error on the $\mathrm{NH}_{3}$ measurement below $0.1 \mathrm{ppb}$ $\left(0.04 \mathrm{ppb}\right.$ for a $50 \%$ relative humidity at $\left.20^{\circ} \mathrm{C}\right)$. Without compensation, a $1.5 \%$ concentration of water (corresponding to a relative humidity of $50 \%$ at $24^{\circ} \mathrm{C}$ ) produces an interferent PA signal equivalent to $38 \mathrm{ppb}$ of ammonia. The quadratic behaviour of the water absorption is clearly seen in Fig. 6.

\subsection{Measurements at low ammonia concentrations}

\subsubsection{Ammonia reference gases}

The strong interaction of $\mathrm{NH}_{3}$ with many surfaces makes the production, storage and use of ammonia calibration gases difficult. This is especially true at low mixing ratios needed for ambient measurements, which stimulated various concepts for the production and use of adequate references gases $[27,28]$. For this study, a continuous gas generator was used,

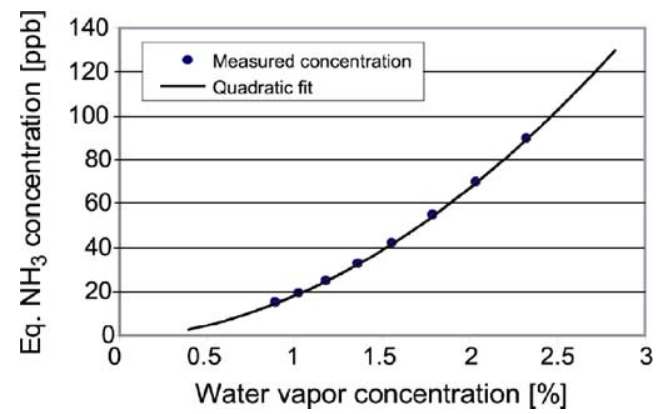

Fig. 6. Contribution of water vapour on the PA signal (in equivalent $\mathrm{NH}_{3}$ concentration). The quadratic behaviour is characteristic of the water vapour continuum absorption.

which is based on the dynamic evaporation of an aqueous ammonia solution into a continuous nitrogen gas stream [29]. A schematic representation of this calibration gas generator is shown in Fig. 7. The liquid is stored in a gas tight bag, which is continuously weighted with an accuracy of $0.1 \mathrm{mg}$, and transported by means of a peristaltic pump to an evaporator kept at $180^{\circ} \mathrm{C}$. Since the carrier gas is controlled by a mass flow controller, both liquid and gas flows have mass units and are, therefore, independent of temperature and pressure.

\subsubsection{Linearity at low $\mathrm{NH}_{3}$ concentrations}

The linearity of the TGA320 was tested for ammonia concentrations between 0 and $100 \mathrm{ppb}$. The calibration gas with variable ammonia concentrations was generated according to the method described in Section 3.3.1. Fig. 8 shows the course of the calibration gas concentration versus time as well as the response of the TGA320. The ammonia concentration in the calibration gas can be changed almost instantaneously. On the other hand, it takes about 20-30 min after a significant increase of the ammonia concentration in the calibration gas until the TGA320 signal is constant. This is very likely due to the polar nature of ammonia, i.e. the time needed to equilibrate adsorption and desorption effects in the tubes and in the measuring cell of the instrument. The measuring principle itself enables a very fast detection of ammonia.

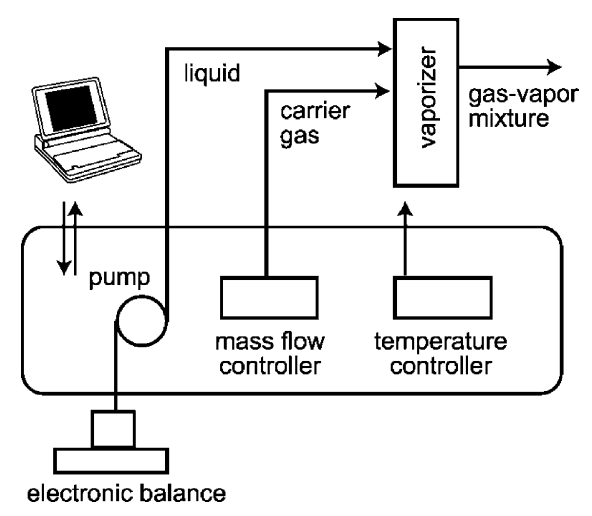

Fig. 7. Schematic representation of the calibration gas generator. 

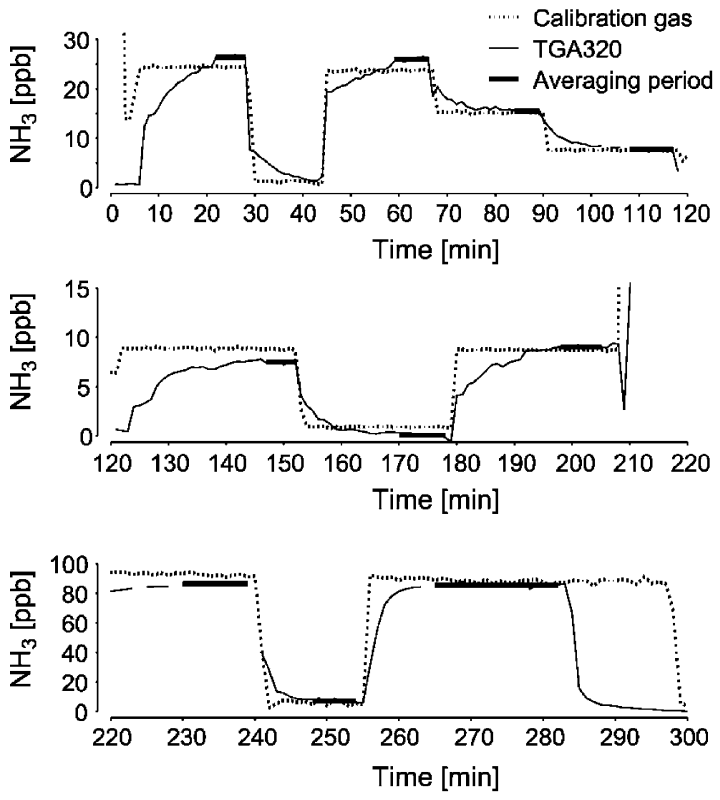

Fig. 8. Course of the $\mathrm{NH}_{3}$ calibration gas and response of the TGA320. The thick black lines indicate the time periods for which the TGA320 signal was taken as constant and averaged for testing the linearity of the instrument (Fig. 9). After $283 \mathrm{~min}$, the TGA320 was detached from the calibration gas supply.

After reaching a constant level, the signal of the TGA320 was averaged for the time period as indicated in Fig. 8. Fig. 9 shows the average instrument reading versus the calibration gas concentration. The response of the TGA320 is almost perfectly linear. This is a remarkable result considering the fact that the calibration measurements were done after 2 months of operation without calibrating the instrument and without zero point adjustments.

\subsection{General performances}

From the user's perspective, a monitoring technique differs mainly by the following features: sampling methods, selectivity, detection limit or sensitivity and user-friendly op-

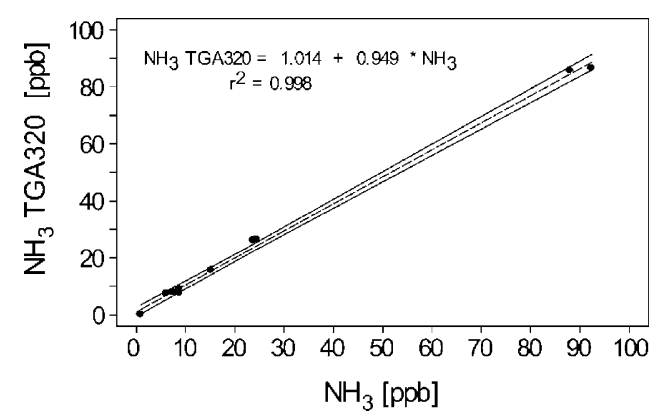

Fig. 9. Linearity of the TGA320 at low $\mathrm{NH}_{3}$ concentrations. Shown is the signal of the instrument vs. the ammonia concentration in the supplied calibration gas. The circles are mean values over the time periods indicated by the thick lines in Fig. 8 . The broken line is the linear regression curve, the two remaining lines are the upper and lower limits of the $95 \%$ confidence band. eration. In industrial processes control, a continuous uninterrupted monitoring is of prime importance. Therefore, in the design of a trace gas monitoring system, the response time and the long-term stability have to be emphasised. PA-based monitoring systems for the detection of ammonia have the advantage to offer low detection limit and continuous sampling method with high flow rates. The TGA300 Series presents the following features and performances:

- Measurement range: $0.1 \mathrm{ppb}-3 \mathrm{ppm}$;

- Detection limit: $0.1 \mathrm{ppb}$;

- Linearity: $r^{2}>0.998$

- Accuracy: $0.25 \mathrm{ppb}$ or $1 \%$;

- Continuous sampling with air flow rate up to $5 \mathrm{l} / \mathrm{min}$.

The excellent linearity and large dynamic range enable a one point calibration at a relatively high concentration value, leading to an accuracy that can be better than $1 \%$ (depending on the standard gas quality).

\section{Examples of applications}

\subsection{Applications in the semiconductor Industry}

\subsection{1. $\mathrm{NH}_{3}$ monitoring in deep UV photolithography}

One of the most powerful driving forces in semiconductor manufacturing has been the continuing trend towards micro-miniaturisation. As semiconductor device geometry and size continue to decrease into the deep sub-micron level, the importance of chemical contamination has become as important as the particle contamination or even more critical. Furthermore, photolithography, the core technology of semiconductor device manufacturing, has dramatically changed as the dimension of the imprinted patterns shrunk to less than $0.25 \mu \mathrm{m}$. Since the photolithography resolution (smallest dimension that can be imprinted on a wafer) is proportional to the exposing wavelength, higher resolution has been achieved by reducing the exposing wavelength. Several photoresist modifications were required to shift from 436 to $300 \mathrm{~nm}$ in order to improve its absorption characteristics. Further reduction to the so-called deep UV region ( $254 \mathrm{~nm}$ and below) necessitated the development of a completely new concept of photosensitive resists in order to overcome the intrinsic sensitivity limitations associated to the reduction of the wavelength: the chemically amplified resist (CAR). In CAR systems, an acid generated by irradiation induces a cascade of chemical transformations, providing a gain mechanism that compensates for the reduction of the resist sensitivity. In other words, a small number of photo-generated acid molecules initiates a catalytic reaction that leads to the transformation of the irradiated area. To-date, CAR has enabled the breakthrough of the now widely used 248, 193 and $157 \mathrm{~nm}$ technologies (sub 0.15- $\mu \mathrm{m}$ linewidth).

As CAR has become very commonly used, new problems of molecular contaminations have appeared in production 
environments in relation with the required chemical amplification process. Any basic impurity which reduces the activity of the photo-generated acid can inhibit the chemical amplification and, consequently, has a dramatic effect on the lithographic performances. As a result, the level of basic impurities is already critical at very low concentrations down to the ppb level or even below. Ammonia has rapidly been identified as one of the dominant basic substances among the airborne molecular contamination (AMC) [30]. Ammonia is the most abundant alkaline component in the atmosphere, it is very volatile and several sources are unavoidable in cleanrooms, including cleaning solutions, materials, human beings, outdoor pollution and several manufacturing processes. For instance, $0.13 \mu \mathrm{m}$ technology requires an ammonia concentration below $1 \mathrm{ppb}$ to guarantee a default free production. Chemical filters have been especially developed in order to fight this contamination problem, but they are unable to completely remove the AMC since the contamination may be generated inside the cleanest areas downward from the filters. Therefore, continuous control of the level of AMC at different locations is increasingly being performed in cleanrooms and new measuring tools are required to perform an efficient detection of $\mathrm{AMC}$ at trace level, and especially of ammonia. While the level of AMC has traditionally been controlled by chromatographic techniques that are not compatible with an on-line continuous monitoring, PAS can offer an extremely efficient tool to detect ammonia at the ppb level. Furthermore, a portable instrument has the advantage to actively trace contamination sources.

\subsubsection{Clean rooms measurements}

One of the unavoidable source of contamination in cleanrooms is the presence of workers (ammonia is found in perspiration and the ammonia concentration in human exhalation exceeds $100 \mathrm{ppb}$ and may even reach $1000 \mathrm{ppb}$ for smokers). Fig. 10 shows typical daily variations of the ammonia concentration measured with the TGA320 in area without chemical filters. The ammonia concentration is seen

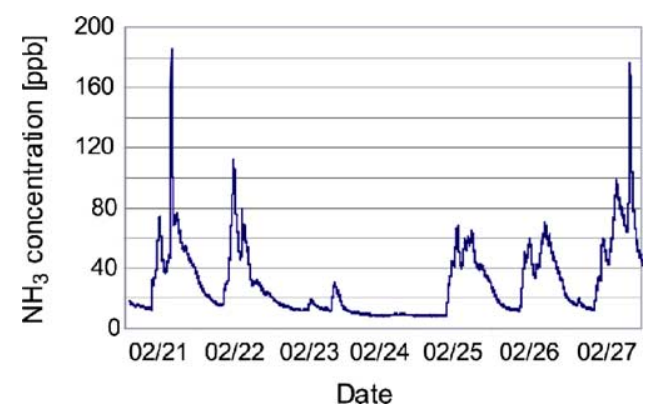

Fig. 10. Daily evolution (Thursday February 21, 2002 to Wednesday February 27, 2002) of the ammonia concentration measured with the TGA320 in a cleanroom (in area not equipped with chemical filters), showing the importance of the internal contamination (staff activity). Over the weekend, the contamination level drops, whereas it peaks in the morning and in the afternoon of weekdays. to be much lower during night-time than day-time, when staff members are more active than in the night shift. Even in closed track environments equipped with chemical filters, peaks of ammonia can be observed when maintenance work is being done in one of the adjacent track, as shown in Fig. 11.

\subsection{Environmental monitoring}

\subsection{1. $\mathrm{NH}_{3}$ in the environment}

The role of ammonia as an atmospheric air pollutant has been recognised for some time. Ammonia in the atmosphere is mainly a result of human activities, in particular agricultural practices. About $80-95 \%$ of the total ammonia emissions in Europe is of agricultural origin, the remainder is attributed to industrial sources, households, pet animals and natural ecosystems [31]. Although there is a large variation from country to country, about $80 \%$ of the agricultural ammonia emissions in Europe are attributed to contribution from animal manure, the remaining $20 \%$ are assigned to the use of fertilisers. The contribution of road traffic to the non-agricultural ammonia emissions has been considered to be rather small. However, ammonia emissions from traffic are expected to be increasing since the introduction of vehicles equipped with catalytic converters [32,33]. Consequently, road traffic might be an important source of ammonia in urban areas [34].

Gaseous ammonia has a relative short atmospheric lifetime and is deposited fairly close to its source [35]. The combination of large variations in emission density, low emission heights, and the high deposition velocity lead to ambient ammonia concentrations that are highly variable on small spatial scales. When dissolved in water droplets, ambient ammonia can form secondary aerosol particles, such as ammonium nitrate and ammonium sulfate, which may remain airborne for longer times and, therefore, can be transported over long distances [36]. Deposition of gaseous ammonia and ammonium aerosol through dry and wet deposition contributes to both acidification and eutrophication, which may cause damage to sensitive ecosystems such as forests, heathlands and bogs. In Switzerland, the deposition of nitrogen

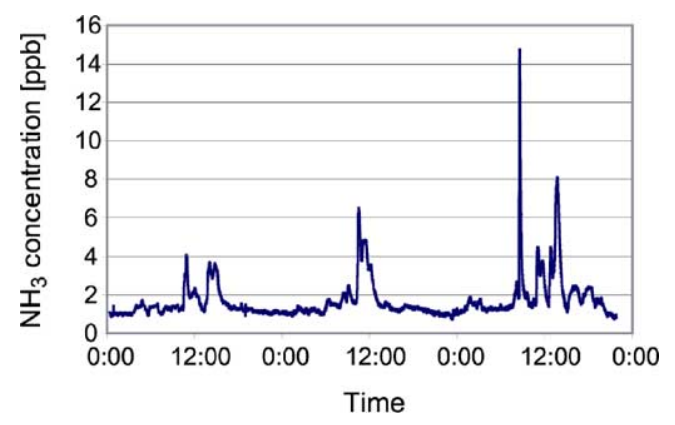

Fig. 11. Ammonia concentration measured with the TGA320 in a clean track environment equipped with chemical filters. Maintenance activity in an adjacent track can generate non-negligible ammonia peaks. 
(from $\mathrm{NO}_{x}$ and $\mathrm{NH}_{3}$ ) exceeds the critical loads for forests in about $90 \%$ of the forest land [37]. In order to account for the complex impacts of ammonia to the environment, reduction targets for ammonia were set by the protocol to the Convention on Long-Range Transboundary Air Pollution in Europe. The member countries of the Convention accepted an obligation to reduce the ammonia emissions by 2010 to the agreed national emission ceilings.

To control the success of reduction measures, accurate and reliable measurement systems for the observation of ambient ammonia levels are required. It is necessary to have instruments available that fulfil the requirements for unattended long-term monitoring, i.e. they should enable user-friendly operation with long maintenance-free intervals, sufficient zero and span point stability, and automatic zero and span point calibrations. PA systems certainly have the potential to fulfil these requirements.

\subsubsection{Ambient air measurement}

Field measurements have been performed at the rural and traffic related monitoring site in the Rhone valley near Sion, Switzerland. The site belongs to the Swiss national air quality monitoring network (NABEL). It is located within $50 \mathrm{~m}$ distance to a motorway with a traffic density of about 20,000 vehicles per day. Fig. 12 shows the TGA310's signal during 4 days in July 2002. The ammonia concentration has a distinct diurnal pattern that is clearly correlated with the traffic density on the nearby motorway (also shown in Fig. 12). These observations indicate that road traffic was the dominating emission source contributing to the ambient ammonia level at the monitoring site during the measuring period.
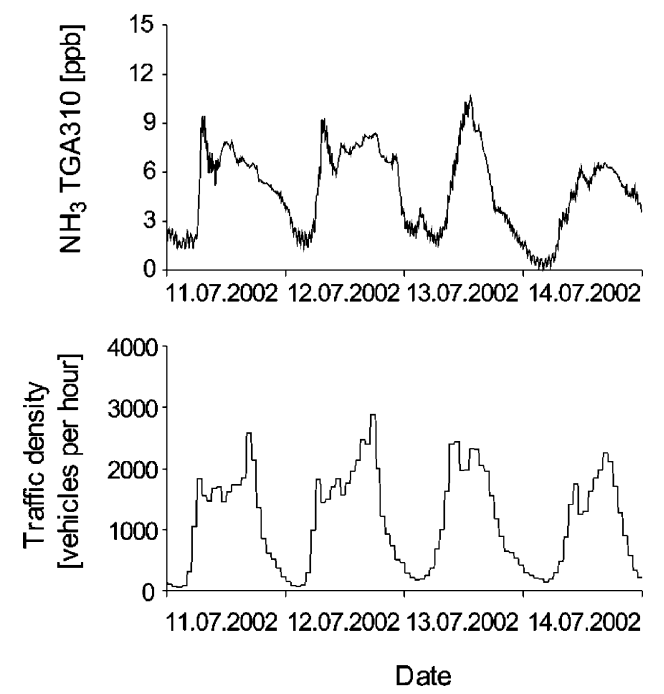

Fig. 12. Four days (Thursday July 11, 2002 to Sunday July 14, 2002) of continuous ammonia measurements at the rural and traffic related site near Sion, Switzerland, obtained with the TGA310 (upper graph). The signal is clearly related to the traffic density on the nearby motorway (lower graph).

\subsubsection{Tunnel study}

Tunnel studies are well suited to determine ammonia emissions, because vehicles are typically operated under hot and stable conditions, which are relevant for $\mathrm{NH}_{3}$ formation in the three way catalyst [34]. In a recent study at the Gubrist Tunnel near Zürich, Switzerland, special care was taken to validate the employed analytical methods. The data shown here compares the TGA310 with FTIR and the Airrmonia monitor (Mechatronics, Netherlands). Furthermore, $24 \mathrm{~h}$ mean values are related to values obtained by the absorption of $\mathrm{NH}_{3}$ in impingers, which represents a well-established averaging technique. Detailed results of the full validation campaign will be published separately.

FTIR measurements were performed with a Nicolet Avatar 370 spectrometer using a Foxboro multipass cell with a $20 \mathrm{~m}$ pass length. Quantitation was done by classical least square fitting using a set of calibration spectra that were measured with the identical optical equipment in the laboratory. Daily mean values were obtained using $30 \mathrm{ml}$ impingers with a nominal flow rate of $21 / \mathrm{min}$ that were filled with 0.001 or $0.01 \mathrm{M} \mathrm{H}_{2} \mathrm{SO}_{4}$. Subsequent analysis was done by ion chromatography. The Airrmonia is based on the absorption of $\mathrm{NH}_{3}$ in an aqueous buffer solution, selective diffusion through a membrane
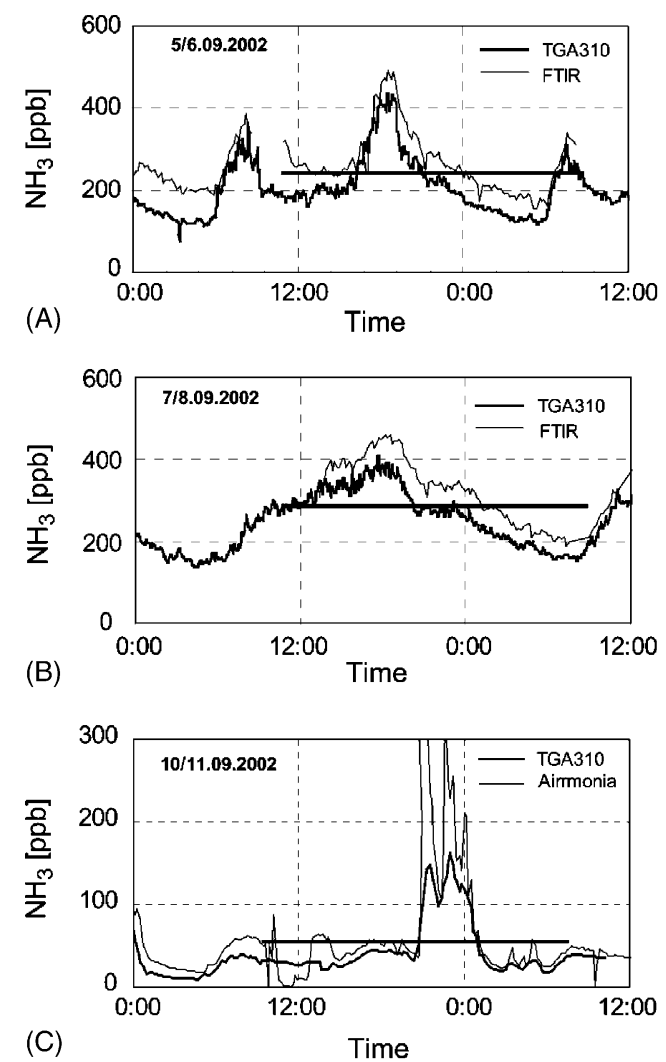

Fig. 13. Measurements of $\mathrm{NH}_{3}$ during a tunnel study at the exit on weekdays (A) and weekends (B), and at much lower concentrations at the entrance during a weekday (C). Data from the TGA310 are compared with FTIR and Airrmonia monitor. Twenty-four-hour average values obtained by absorption in impingers are also represented as straight lines. 
into high purity water, and measurement of the changes in conductivity.

Some examples of the data obtained are shown in Fig. 13. The concentration pattern with two peaks on weekdays (A) and one broader peak on weekends (B) reflects the typical traffic intensity. The trends and overall concentrations of TGA310 and FTIR agree well. Higher concentrations after peak values can be interpreted as tailing of the FTIR which indicates adsorption onto the walls of the measurement cell. Measurement interruptions are due to maintenance and recalibration. At the tunnel entrance, much lower concentrations are found (C). Comparison of the TGA310 with the Airrmonia shows reasonable agreement at low concentrations and very pronounced differences for values above $100 \mathrm{ppb}$. The latter are due to signals that are significantly out of the Airrmonia's non-linear calibration. The impinger measurements are given as a straight line representing the sampling period and the mean value. The average data of the TGA310 correspond to $83-95 \%$ of the impinger measurements $(N=7)$ at the exit, and to $93-97 \%(N=2)$ at the tunnel entrance.

\section{Conclusions}

A portable ammonia analyser based on laser PAS has been developed and tested according to industry and environmental requirements. A singlemode $\mathrm{CO}_{2}$ laser has been used for the PA excitation, while the acoustic detection has fully taken advantage of the high sensitivity offered by a resonant configuration. The resonant cell has been designed to maximize the PA excitation of the first radial mode in the $10 \mathrm{kHz}$ range. A quality factor as high as 780 has been obtained with a diagonal beam excitation and an axial microphone position guarantees the detection of the generated acoustic wave in the best conditions, leading to a detection limit below $0.1 \mathrm{ppb}$ of ammonia. The laser wavelength has been carefully selected in order to achieve strong ammonia absorption, while keeping the possible interferences from other species as low as possible. Among all the atmospheric constituents, only water vapour contributes in a non-negligible way to the generated PA signal, due to its broadband absorption in the whole $\mathrm{CO}_{2}$ laser spectrum range, and consequently needs to be compensated. Therefore, a high performance hygrometer has been integrated in the flow circuit in order to precisely and continuously determine the water vapour concentration in the measured air samples and the corresponding contribution is subtracted from the measured PA signal. As a result, the total interference arising from atmospheric constituents, including water vapour, is kept below the detection limit, i.e. lower than $0.1 \mathrm{ppb}$. The large dynamic range, from $0.1 \mathrm{ppb}$ to more than $3 \mathrm{ppm}$, and the excellent linearity $\left(r^{2}>0.998\right)$ enable a precise calibration of the system with a single standard gas at a relatively high concentration, which guarantees a high accuracy at very low levels as well as at high concentrations. Since response time and continuous automatic monitoring are important features in actual applications, additional bumpers have been placed at the gas inlet and outlet in order to enable measurements at high flow rates, up to $5 \mathrm{l} / \mathrm{min}$. Measurement capability at high flow rates has demonstrated to be of prime importance in the case of ammonia monitoring, because of the adsorption/desorption effects due to the polar characteristic of the $\mathrm{NH}_{3}$ molecule, which makes its monitoring at trace level extremely difficult. Only flow rates larger than $11 / \mathrm{min}$ can guarantee an unbiased measurement and can offer the required short response time of most industrial applications.

A dedicated DSP-based electronics has been developed for the processing of the detected acoustic signals and the computation of the ammonia concentration in real-time. The electronics also includes continuous frequency tracking and real-time water vapour compensation, which makes the system totally autonomous. A computer interfaces directly with the system, updates the measured concentration value every $3 \mathrm{~s}$ and enables unattended automatic measurements.

The system performances and operation have been tested in actual conditions in cleanrooms environments. The sensitivity and response time have demonstrated to be totally compatible with the application requirements. The portability of the monitoring system makes it a very efficient tool for contamination control in photolithographic facilities, where ammonia is critical as soon as its concentration exceeds $1 \mathrm{ppb}$.

Finally, ammonia has become an important compound in the overall atmospheric pollution and new measuring equipments are required in order to perform long-term continuous monitoring of this species. In addition to sensitivity below the ppb level, long-term stability and simple calibration are fundamental requirements for environmental monitoring. The developed system has been tested both in a road tunnel study, where concentrations in the $100 \mathrm{ppb}$ range were regularly measured, and in rural areas where the measured concentrations were much lower. In each case, the data were in good agreement with other measuring methods over several weeks without recalibration of the system.

\section{References}

[1] A.G. Bell, Am. J. Sci. 20 (1880) 305-324.

[2] A.G. Bell, Philos. Mag. 11 (5) (1881) 510.

[3] I.G. Calasso, V. Funtov, M.W. Sigrist, Appl. Opt. 36 (15) (1997) 3212-3216.

[4] A. Thöny, M.W. Sigrist, Infrared Phys. Technol. 36 (2) (1995) 585615.

[5] C. Brand, A. Winkler, P. Hess, A. Miklos, Z. Bozoki, J. Sneider, Appl. Opt. 34 (18) (1995) 3257-3266.

[6] R. Gerlach, N.M. Amer, Appl. Phys. 23 (1980) 319-326.

[7] M. Wolff, H. Harde, Infrared Phys. Technol. 41 (2000) 283-286.

[8] A. Petzold, R. Niessner, Appl. Phys. Lett. 66 (10) (1995) 1285-1287.

[9] Z. Bozóki, A. Mohácsi, G. Szabó, Z. Bor, M. Erdélyi, W. Chen, F.K. Tittel, Appl. Spectosc. 56 (6) (2002) 715-719.

[10] A. Boschetti, D. Bassi, E. Iacob, S. Ionnatta, L. Ricci, M. Scotoni, Appl. Phys. B 74 (2002) 273-278. 
[11] J. Sneider, Z. Bozóki, A. Miklos, Z.S. Bor, G. Szabó, Int. J. Environ. Anal. Chem. 67 (1997) 253-260.

[12] S. Barbieri, J.-P. Pellaux, E. Studemann, D. Rosset, Rev. Sci. Instrum. 73 (6) (2002) 2458-2461.

[13] V.A. Kapitanov, Yu.N. Ponomarev, K. Song, H.K. Cha, J. Lee, Appl. Phys. B 73 (2001) 745-750.

[14] B.A. Paldus, T.G. Spence, R.N. Zare, J. Oomens, F.J.M. Harren, D.H. Parker, C. Gmachl, F. Cappasso, D.L. Sivco, J.N. Baillargeon, A.L. Hutchinson, A.Y. Cho, Opt. Lett. 24 (3) (1999) 178-180.

[15] L.B. Kreuzer, in: Y.-H. Pao (Ed.), Optoacoustic Spectroscopy and Detection, Academic Press, New York, 1977, Chapter 1, pp. 1-25.

[16] A. Miklos, S. Schäfer, P. Hess, in: J.C. Lindon, G.E. Tranter, J.L. Homes (Eds.), Encyclopedia of Spectroscopy and Spectrometry, Academic Press, San Diego, 2000, pp. 1815-1822.

[17] A. Miklos, P. Hess, Z. Bozoki, Rev. Sci. Instrum. 72 (4) (2001) 1937-1955.

[18] J.P.M. Trusler, Physical Acoustics and Metrology of Fluids, Adam Hilger, Bristol, 1991.

[19] A.C. Tam, Rev. Mod. Phys. 58 (2) (1986) 381-431.

[20] W. Faubel, in: A. Mandelis, P. Hess (Eds.), Life and Earth Sciences, SPIE, Bellingham, Washington, 1997, Chapter 8, pp. 289-328.

[21] C.F. Dewey, in: Y.-H. Pao (Ed.), Optoacoustic Spectroscopy and Detection, Academic Press, New York, 1977, Chapter 3, pp. 47-77.

[22] L.-Y. Hao, J.-X. Han, Q. Shi, J.-H. Zhang, J.-J. Zheng, Q.-S. Zhu, Rev. Sci. Instrum. 71 (5) (2000) 1975-1980.

[23] A. Schmohl, A. Miklos, P. Hess, Appl. Opt. 40 (15) (2001) 25712578.

[24] M.W. Sigrist, Infrared Phys. Technol. 36 (1) (1995) 415-425.
[25] M.E. Thomas, R.J. Nordstrom, J. Quantum Spectrosc. Radiat. Transfer 28 (2) (1982) 81-101.

[26] J.S. Ryan, M.H. Hubert, R.A. Crane, Appl. Opt. 22 (5) (1983) $711-$ 717.

[27] F.C. Fehsenfeld, L.G. Huey, E. Leibrock, R. Dissly, E. Williams, T.B. Ryerson, R. Norton, D.T. Sueper, B. Hartsell, J. Geophys. Res.-Atmos. 107 (D24) (2002) 2801-2814.

[28] J.A. Neuman, T.B. Ryerson, L.G. Huey, R. Jakoubek, J.B. Nowak, C. Simons, F.C. Fehsenfeld, Environ. Sci. Technol. 37 (13) (2003) 2975-2981.

[29] P. Wilbing, M. Schmäh, International Conference on Emissions Monitoring, Arnhem, Netherlands, 2001.

[30] K.R. Dean, D.A. Miller, R.A. Caprio, J.S. Petersen, G.K. Rich, J. Photopolym. Sci. Technol. 10 (3) (1997) 425-444.

[31] K.W. Van der Hoek, Atmos. Environ. 32 (3) (1998) 315-316.

[32] M.A. Moeckli, M. Fierz, M.W. Sigrist, Environ. Sci. Technol. 30 (9) (1996) 2864-2867.

[33] M.A. Sutton, U. Dragositis, Y.S. Tang, D. Fowler, Atmos. Environ. 34 (6) (2000) 855-869.

[34] C. Perrino, M. Catrambone, A.D.M. Di Bucchianico, I. Allegrini, Atmos. Environ. 36 (34) (2002) 5385-5394.

[35] W.A.H. Asman, Atmos. Environ. 32 (3) (1998) 415-421.

[36] E. Berge, J. Bartnicki, K. Olendrzynski, S.G. Tsyro, J. Environ. Manag. 57 (1) (1999) 31-50.

[37] BUWAL, Ammoniak ( $\left.\mathrm{NH}_{3}\right)$-Minderung bei der landwirtschaftlichen Nutztierhaltung, Mitteilungen zur Luftreinhalte-Verordnung Nr. 13, Bern, Schweiz, 2002. 